\title{
Principales intoxications végétales chez les ruminants en zone méditerranéenne
}

\author{
D. Mohammedi ${ }^{1 *}$ S. Mohammedi ${ }^{1}$ G. Keck ${ }^{2}$
}

\begin{abstract}
Mots-clés
Ruminant - Empoisonnement Hyoscyamus muticus - Ferula communis - Quercus coccifera Cistus monspeliensis - Silybum marianum - Algérie.
\end{abstract}

\begin{abstract}
Résumé
Les intoxications végétales chez les ruminants sont assez fréquentes mais elles restent méconnues et d'importance difficile à quantifier du fait de la diversité des plantes toxiques qui en sont responsables. Les impacts des intoxications végétales dans le Bassin méditerranéen devraient être mieux évalués pour les distinguer de ceux causés par des infections. En effet, les vétérinaires établissent difficilement un diagnostic d'intoxication végétale et incriminent souvent les agents infectieux. Il est donc important de réunir un maximum d'informations objectives, ce qui est souvent assez délicat au regard des informations relativement approximatives dans les descriptions des cas. Certaines plantes responsables d'intoxication ont une répartition très large au sud et au nord de la Méditerranée, y compris en France. D'autres sont au contraire relativement spécifiques des milieux désertiques du Maghreb et sont retrouvées notamment en Algérie. Les animaux les plus fréquemment concernés sont les ovins (61,5 p. 100), les bovins (24,7 p. 100), les dromadaires (9 p. 100) et les caprins (4,6 p. 100). Les intoxications rencontrées chez les ruminants sont provoquées par Hyoscyamus muticus spp. falezlez (Coss.) Maire, Ferula communis L., Thapsia garganica, Hypericum crispum L., Tetraena cornuta (Coss.) Beier \& Thulin, Drimia maritima (L.) Stearn, Quercus coccifera L., Nerium oleander L., Cistus monspeliensis L., Silybum marianum (L.) Gaertn., Sorghum drummondii (Stend.) Nees ex Millsp. \& Chase, Pteridium aquilinum (L.) Kuhn, Malva parviflora L., Oxalis pes-caprae L., Sorghum halepense (L.) Pers., Calobota saharae, Diplotaxis harra (Forssk.) Boiss.
\end{abstract}

\section{INTRODUCTION}

Les plantes toxiques sont nombreuses. Toutefois, leur incrimination dans les cas de mortalités d'animaux est difficile à établir avec certitude. Il est souvent difficile de poser un diagnostic d'intoxication végétale d'autant que c'est un argument facilement avancé par les éleveurs lors de pathologies non élucidées. L'identification de la plante incriminée n'est pas toujours aisée, les connaissances botaniques, notamment des vétérinaires, étant souvent insuffisantes. Il n'est pas aisé d'évaluer la quantité et le type de

\footnotetext{
1. Ecole nationale supérieure vétérinaire d'Alger, rue Assad Abbés, El Alia, Alger, Algérie.

2. Ecole nationale vétérinaire de Lyon, Unité de pharmacie toxicologie, 69280 Marcy l'Etoile, France.

* Auteur pour la correspondance

E-mail : mohammedidahmane@yahoo.fr
}

végétaux toxiques consommés, et de confirmer la relation entre leur consommation et les signes cliniques observés.

L'objectif du présent travail a été de répertorier les plantes toxiques les plus courantes dans les différentes régions d'Algérie afin de les faire connaître aux vétérinaires, ce qui leur permettrait de les inclure dans leur diagnostic différentiel et, dans la mesure du possible, de donner des conseils aux éleveurs en terme de prévention et pour une meilleure prise en charge lors d'une intoxication végétale.

\section{MATERIEL ET METHODES}

Les données épidémiologiques dont nous disposons sont basées sur les informations fournies par les vétérinaires du service de toxicologie de l'Ecole nationale vétérinaire d'Alger. Ces vétérinaires sont environ une centaine, installés dans différentes régions du pays. Ceux qui interviennent auprès de plusieurs types d'animaux (ruminants, carnivores et volailles) ou auprès de ruminants 
uniquement sont minoritaires par rapport à ceux qui sont consultés seulement pour les volailles. Par conséquent, les déclarations de cas d'intoxication végétale sont peu nombreuses.

La première étape de cette enquête épidémiologique a été de collecter les informations, puis d'établir des hypothèses. La deuxième étape a été l'évaluation du risque en fonction de la toxicité de la plante suspectée, des quantités ingérées et des circonstances décrites. Ces informations ont permis de vérifier (ou non) les hypothèses initiales faites par le vétérinaire, en fonction des données bibliographiques et de l'expertise progressivement mise en place.

\section{RESULTATS ET DISCUSSION}

D'après les données issues d'appels et de cas transmis, les végétaux ont représenté la deuxième cause d'intoxication (14 à

\section{Tableau I}

Nombre de cas ${ }^{*} \mathrm{~d}^{\prime}$ intoxication par espèce animale et végétale en Algérie de 1995 à 2013

\begin{tabular}{|c|c|c|c|c|}
\hline Plante & Ovin & Bovin & Caprin & Dromadaire \\
\hline $\begin{array}{l}\text { Hyoscyamus muticus } \\
\text { spp. falezlez (Coss.) } \\
\text { Maire (jusquiame falezlez) }\end{array}$ & 12 & 0 & 10 & 15 \\
\hline Thapsia garganica L. & 20 & 0 & 0 & 12 \\
\hline $\begin{array}{l}\text { Hypericum crispum L. } \\
\text { (millepertuis) }\end{array}$ & 28 & 0 & 0 & 0 \\
\hline $\begin{array}{l}\text { Tetraena cornuta (Coss.) } \\
\text { Beier \& Thulin (zygophylle) }\end{array}$ & 35 & 0 & 0 & 0 \\
\hline $\begin{array}{l}\text { Nerium oleander } \mathrm{L} \text {. } \\
\text { (laurier rose) }\end{array}$ & 0 & 0 & 12 & 0 \\
\hline $\begin{array}{l}\text { Cistus monspeliensis L. } \\
\text { (ciste de Montpellier) }\end{array}$ & 0 & 60 & 0 & 0 \\
\hline $\begin{array}{l}\text { Silybum marianum (L.) } \\
\text { Gaertn. (chardon-Marie) }\end{array}$ & 0 & 50 & 0 & 0 \\
\hline $\begin{array}{l}\text { Sorghum drummondii } \\
\text { (Stend.) Nees ex Millsp. } \\
\text { \& Chase (sorgho) }\end{array}$ & 0 & 12 & 0 & 0 \\
\hline $\begin{array}{l}\text { Pteridium aquilinum (L.) } \\
\text { Kuhn (fougère aigle) }\end{array}$ & 0 & 10 & 0 & 0 \\
\hline Malva parviflora L. (mauve) & 0 & 12 & 0 & 12 \\
\hline $\begin{array}{l}\text { Ferula communis L. } \\
\text { (férule commune) }\end{array}$ & 150 & 25 & 0 & 0 \\
\hline Oxalis pes-caprae L. (oxalis) & 0 & 35 & 0 & 0 \\
\hline $\begin{array}{l}\text { Sorghum halepense (L.) } \\
\text { Pers. (sorgho d'Alep) }\end{array}$ & 0 & 0 & 0 & 12 \\
\hline $\begin{array}{l}\text { Calobota saharae } \\
\text { (genêt saharien) }\end{array}$ & 0 & 0 & 0 & 15 \\
\hline $\begin{array}{l}\text { Diplotaxis harra } \\
\text { (Forssk.) Boiss. }\end{array}$ & 0 & 0 & 0 & 15 \\
\hline $\begin{array}{l}\text { Drimia maritima (L.) } \\
\text { Stearn. (scille maritime) }\end{array}$ & 25 & 24 & 0 & 0 \\
\hline $\begin{array}{l}\text { Quercus coccifera L. } \\
\text { (chêne kermès) }\end{array}$ & 22 & 20 & 0 & 0 \\
\hline Total des cas & \multicolumn{4}{|c|}{643} \\
\hline
\end{tabular}

18 p. 100) chez les animaux, après les pesticides agricoles (45 à 50 p. 100). Les ovins ont été l'espèce la plus touchée.

De 1995 à 2013, nous avons recensé 643 cas collectifs d'intoxications végétales en Algérie (tableau I). Plus de 3000 animaux ont été affectés par ces intoxications végétales car un cas peut représenter plusieurs animaux, parfois jusqu'à une centaine (tableau II), contrairement aux carnivores où une déclaration correspond à un individu. Les espèces les plus fréquemment concernées ont été les ovins $(61,5$ p. 100$)$ et les bovins $(24,7$ p. 100$)$, puis les dromadaires ( 9 p. 100) et les caprins $(4,6$ p. 100) (tableau II). La répartition des intoxications en fonction des espèces végétales est indiquée dans le tableau II. La répartition par famille de plantes a été différente pour chaque espèce animale (tableau I). En fonction de l'espèce affectée, les caractéristiques géographiques, physiques et toxicologiques des plantes, et/ou les symptômes et les lésions qu'elles ont causés sont décrits ci-après.

\section{Tableau II}

Nombre d'intoxications par espèce animale et végétale en Algérie de 1995 à 2013

\begin{tabular}{|c|c|c|c|c|}
\hline Plante & Ovin & Bovin & Caprin & Dromadaire \\
\hline $\begin{array}{l}\text { Hyoscyamus muticus } \\
\text { spp. falezlez (Coss.) } \\
\text { Maire (jusquiame falezlez) }\end{array}$ & 1023 & 0 & 138 & 60 \\
\hline Thapsia garganica & 170 & 0 & 0 & 36 \\
\hline $\begin{array}{l}\text { Hypericum crispum L. } \\
\text { (millepertuis) }\end{array}$ & 160 & 0 & 0 & 0 \\
\hline $\begin{array}{l}\text { Tetraena cornuta (Coss.) } \\
\text { Beier \& Thulin (zygophylle }\end{array}$ & le) 120 & 0 & 0 & 0 \\
\hline $\begin{array}{l}\text { Nerium oleander L. } \\
\text { (laurier rose) }\end{array}$ & 0 & 0 & 10 & 0 \\
\hline $\begin{array}{l}\text { Cistus monspeliensis L. } \\
\text { (ciste de Montpellier) }\end{array}$ & 0 & 220 & 0 & 0 \\
\hline $\begin{array}{l}\text { Silybum marianum (L.) } \\
\text { Gaertn. (chardon-Marie) }\end{array}$ & 0 & 56 & 0 & 0 \\
\hline $\begin{array}{l}\text { Sorghum drummondii } \\
\text { (Stend.) Nees ex Millsp. } \\
\text { \& Chase (sorgho) }\end{array}$ & 0 & 38 & 0 & 0 \\
\hline $\begin{array}{l}\text { Pteridium aquilinum (L.) } \\
\text { Kuhn (fougère aigle) }\end{array}$ & 0 & 45 & 0 & 0 \\
\hline Malva parviflora L. (mauve) & 0 & 50 & 0 & 66 \\
\hline $\begin{array}{l}\text { Ferula communis L. } \\
\text { (férule commune) }\end{array}$ & 300 & 120 & 0 & 0 \\
\hline Oxalis pes-caprae L. (oxalis) & 0 & 120 & 0 & 0 \\
\hline $\begin{array}{l}\text { Sorghum halepense (L.) } \\
\text { Pers. (sorgho d'Alep) }\end{array}$ & 0 & 0 & 0 & 24 \\
\hline $\begin{array}{l}\text { Calobota saharae } \\
\text { (genêt saharien) }\end{array}$ & 0 & 0 & 0 & 36 \\
\hline $\begin{array}{l}\text { Diplotaxis harra (Forssk.) } \\
\text { Boiss. }\end{array}$ & 0 & 0 & 0 & 75 \\
\hline $\begin{array}{l}\text { Drimia maritima (L.) } \\
\text { Stearn. (scille maritime) }\end{array}$ & 125 & 95 & 0 & 0 \\
\hline $\begin{array}{l}\text { Quercus coccifera L. } \\
\text { (chêne kermès) }\end{array}$ & 115 & 66 & 0 & 0 \\
\hline $\begin{array}{l}\text { Total } \\
(\%)\end{array}$ & $\begin{array}{l}2013 \\
(61,6)\end{array}$ & $\begin{array}{c}810 \\
(24,8)\end{array}$ & $\begin{array}{l}148 \\
(4,6)\end{array}$ & $\begin{array}{l}297 \\
(9)\end{array}$ \\
\hline
\end{tabular}




\section{Ovins}

Les intoxications rencontrées chez les ovins ont été provoquées par sept espèces de plantes (tableau II).

\section{Hyoscyamus muticus spp.falezlez (Coss.) Maire (jusquiame, falezlez, bettina, ifelehleh)}

\section{- Caractéristiques}

La jusquiame est une plante herbacée des sables et des pâturages sablonneux sahariens, de la famille des solanacées. Elle est vivace avec des tiges nombreuses dressées, des feuilles lancéolées pétiolées, une corolle blanchâtre, et d'un violet noir dans le haut du tube $(8,13)$.

Elle renferme trois alcaloïdes : l'hyoscyamine, un peu d'atropine et de la scopolamine. L'atropine et l'hyoscyamine sont des esters du tropanol. L'hyoscyamine est la forme lévogyre alors que l'atropine est la forme racémique. La scopolamine, également appelée hyoscine, est lévogyre. Ces alcaloïdes ont des propriétés parasympatholytiques $(9,56)$.

La scopolamine, du point de vue pharmacologique, se différencie nettement de l'atropine. Alors que cette dernière est un excitant central, la scopolamine est un sédatif central. Cette action sur le système central est d'autant plus accentuée que l'encéphale du sujet est développé ; les animaux y sont donc moins sensibles que l'homme. Comme l'atropine, la scopolamine a des propriétés parasympatholytiques.

\section{- Symptômes et lésions}

Les effets spécifiques de l'atropine, qui résultent de son action périphérique, comprennent : l'arrêt de certaines sécrétions (sueur, mucus, salive), l'inhibition du système vagal (tachycardie, dilatation des pupilles et paralysie de l'accommodation du cristallin) et enfin le relâchement des fibres musculaires lisses (bronchiques, intestinales ou autres). Les effets centraux se manifestent par de l'excitation, suivie de dépression et de paralysie du bulbe rachidien.

Dans l'intoxication collective rapportée par les vétérinaires de la région de Béchar, dans le Sud-Ouest algérien, les premiers symptômes sont apparus 24 heures après l'arrivée des animaux au pâturage, et les premiers cas de mortalité 48 heures après. L'intoxication serait liée à la méconnaissance de cette plante par les animaux qui venaient d'une région où elle leur est inconnue. De plus, sur les lieux de pâture, le dessèchement de la flore était presque total, sauf pour cette plante qui présentait des feuilles bien vertes.

Les manifestations de dépression du système nerveux étaient nettes : apathie, prostration, errance (action sédative centrale de la scopolamine). Certains sujets ont présenté une hyperexcitabilité nerveuse et neuromusculaire : agitation et excitabilité, torticolis et signes de démence. De la tachycardie, de la polypnée et un dessèchement total des muqueuses buccales ont été également notés mais paradoxalement environ 40 p. 100 des animaux ont présenté du ptyalisme. Ces symptômes, observés chez les ovins intoxiqués par $H$. muticus spp. dans la région de Béchar, ont été assez proches de ceux rapportés chez des bovins empoisonnés par $H$. niger en Grande Bretagne (16)

Les mortalités ont été de 60 p. 100 dans les troupeaux affectés, ce qui atteste de la toxicité de la jusquiame pour les ovins. A l'autopsie, les vétérinaires ont retrouvé un foie hypertrophié et noirâtre, une vésicule biliaire hypertrophiée et une congestion intestinale. Le contenu des estomacs était desséché. Il y avait également un hydropéricarde.

\section{Ferula communis L. (férule commune, kalkha, toufelt)}

\section{- Caractéristiques}

Ferula communis L., de la famille des Apiaceae (ombellifères), est largement rencontrée dans les pays méditerranéens et particulièrement en Algérie. C'est une grande plante de deux à quatre mètres de haut, à tiges épaisses et creuses. Elle pousse au sud et au nord de la Méditerranée, notamment en Corse où elle est abondante (44). Elle préfère les terrains calcaires, les coteaux arides et les murs, où elle fleurit de juin à août en France, beaucoup plus tôt dans les zones plus arides (mars-avril en Algérie).

La férule contient, surtout au début de la période de végétation et au niveau des racines, un latex toxique provoquant un syndrome hémorragique (36). Le principe toxique serait une substance coumarinique analogue au dicoumarol (14). Les inflorescences non épanouies (boubal) sont consommées cuites, comme légume, bien qu'elles ne soient pas totalement inoffensives (37) : elles pourraient induire des troubles de la coagulation en cas de troubles de l'hémostase préexistants ou chez la femme en période de menstruation (34).

Bien que cette espèce soit toxique pour l'homme, elle est encore plus dangereuse pour les animaux domestiques, notamment les moutons. Au Maroc, deux variétés de Ferula communis sont rencontrées : la variété brevifolia (sur la côte atlantique) et la variété genuina (à l'intérieur du pays) (35). En Algérie, on retrouve la variété brevifolia dans les zones côtières, la variété genuina dans les zones montagneuses et une troisième variété, jamais décrite auparavant, qui semble être un hybride des deux variétés précédentes (42). La férule commune a fait l'objet de nombreuses études également en Italie (4, 5), en Israël (49) et en Espagne (24, 51). Elle est peu étudiée en Algérie bien que la première description de l'intoxication, « le férulisme », ait été faite par une équipe de l'Institut Pasteur d'Alger $(12,55)$. Alors que son feuillage et ses tiges verdoyantes parsèment les pâturages, les animaux évitent de la consommer : ils le font lorsque plus aucun autre fourrage n'est disponible (49).

Les coumarines prénylées, responsables de l'activité anticoagulante de la férule, sont le férulénol (3-farnésyl-4-hydroxycoumarine), la ferprénine et des dérivés proches. Les variations de toxicité de la férule, connues depuis très longtemps, sont principalement liées à une variabilité chimique intraspécifique : les racines des férules sardes contiennent soit des sesquiterpènes, soit des dérivés 4-hydroxycoumariniques (54), le composé majoritaire pouvant être le férulénol ou son dérivé $\omega$-oxygéné (4). Les férules du Maroc ont une composition voisine (férulénol et ses dérivés hydroxylés en C-12' et C-15', isoferprénine) mais, là encore, différente selon la variété $(34,38)$.

\section{- Symptômes et lésions}

L'intoxication des moutons provoque de la prostration et de l'épistaxis. Des diarrhées hémorragiques apparaissent après quelques heures, suivies d'émission de sang non coagulé. Une hématurie est observée, puis l'animal présente de la dyspnée et meurt rapidement. Chez toutes les espèces atteintes, les diarrhées sont présentes. Après les premiers signes d'intoxication, les mortalités sont élevées (75 p. 100).

Dans les cas qui nous ont été décrits, des hémorragies et de l'abattement étaient présents et provenaient de différentes régions du pays, en particulier de la région de Saida. Les vétérinaires ont rapporté une anorexie subite, des diarrhées hémorragiques, une hématurie et une épistaxis. Les animaux se tenaient à l'écart du troupeau, insensibles à leur environnement et prostrés. Ils ont présenté une tachypnée, une tachycardie et un pouls filant. La respiration 
des animaux intoxiqués est devenue de plus en plus dyspnéique à mesure que l'anémie s'installait, puis ils sont tombés et sont morts rapidement. Les mortalités ont été très élevées, proches parfois de 90 p. 100. Les seules lésions post mortem observées ont été une congestion généralisée et d'importantes hémorragies avec hémothorax, hémopéritoine, hématomes et suffusions sous-cutanées. Ces signes cliniques et ces lésions ont également été rapportés par d'autres auteurs $(14,49)$.

\section{Thapsia garganica L. (derias, bounafaa)}

\section{- Caractéristiques}

Thapsia garganica est une plante vivace, de la famille des Apiaceae (ombellifères), à tige florifère dressée, peu ramifiée, haute environ de $1,50 \mathrm{~m}$. Elle a de grandes feuilles en touffe, très découpées, à divisions linéaires, pourvues d'un pétiole en gaine à la base. Les feuilles supérieures sont réduites à une gaine large et épaisse, d'un vert grisâtre comme la tige. Les fruits sont ovales et longs de plus de deux centimètres, et largement ailés. La racine est volumineuse, noirâtre à l'extérieur et blanche à l'intérieur. La résine extraite de la souche a des propriétés vésicantes (50). Les racines contiennent principalement des constituants volatils et des lactones sesquiterpènes, dont la thapsigargine (17). La thapsigargine est capable d'induire la libération d'histamines de diverses cellules de l'organisme. Cette propriété est à l'origine du caractère vésicant mais aussi d'intoxication humaine si elle est consommée (3).

\section{- Symptômes et lésions}

Dans les cas de la région de Djelfa qui nous ont été rapportés, notamment à Birine, les moutons ont consommé des graines séchées de Thapsia. Au début, ils ont présenté des signes d'hyperexcitabilité avec des tremblements, des crises épileptiformes avec écoulement de bave, puis de l'abattement et de la prostration. Ils ont pris une posture d'auto-auscultation avec la tête fortement fléchie sur le thorax. En fin d'évolution, après une phase de paralysie, ils sont entrés dans le coma. Des signes de gastro-entérite et d'hématurie ont été notés.

Cette intoxication a affecté environ 5 à 10 p. 100 des animaux du troupeau, notamment les brebis et surtout les agneaux. Les mâles adultes, engraissés dans d'autres enclos, n'ont pas été concernés par l'intoxication. Les brebis se sont rétablies après une longue convalescence alors que les conséquences de cet empoisonnement sur les agneaux ont été très importantes avec un taux de mortalités de 100 p. 100. Les lésions observées correspondaient à celles d'une gastro-entérite avec un foie blanchâtre. Ces symptômes et ces lésions ont été similaires à ceux rapportés chez les ruminants d'Afrique du Nord (19).

\section{Hypericum crispum L. (millepertuis, el hamra)}

\section{- Caractéristiques}

Le millepertuis est une plante de la famille des clusiacées (Clusiaceae ex. guttifères, hypéricacées), haute de 10 à $40 \mathrm{~cm}$, à tiges herbacées régulièrement ramifiées. Ses feuilles à bords ondulés sont parsemées de nombreux petits points qui sont des glandes contenant les huiles essentielles (41). La fleur est jaune d'or.

Le millepertuis est une plante photosensibilisante qui, consommée par les animaux, provoque un syndrome appelé hypéricisme. Le principe toxique est l'hypéricine, hexa-oxy-2-2'-diméthylnaphtodianthrone, pigment rouge fluorescent responsable des propriétés photosensibilisantes de la plante $(6,29,33,52)$. Les ovins et les bovins sont les espèces les plus fréquemment affectées. Toutes les parties de la plante sont toxiques lorsqu'elles sont ingérées en quantités suffisantes. Les intoxications se produisent lorsque la plante est jeune et qu'elle est dominante dans le pâturage.

\section{- Symptômes et lésions}

Les signes cliniques peuvent apparaitre dans les quelques jours qui suivent le début du pâturage et disparaissent habituellement une à deux semaines après le retrait des animaux (11). Dans les cas décrits en Algérie, autour d'Annaba, des lésions cutanées sont apparues sur les oreilles et sur les régions dépourvues de laine sous forme d'érythème très prurigineux avec grattage intense. Les oreilles étaient enflées et exsudaient un liquide jaunâtre. Ces signes cliniques et ces lésions étaient très semblables à ceux décrits chez des ovins intoxiqués par le millepertuis (16).

\section{Tetraena cornuta (Coss.) Beier \& Thulin (zygophylle, bou griba,} haaka)

\section{- Caractéristiques}

Tetraena cornuta (anciennement Zygophyllum cornutum) est un arbuste de la famille des zygophyllacées, à rameaux blanchâtres et comme emboîtés. Les feuilles comportent deux folioles, les fleurs sont blanchâtres. Les fruits, de couleur violette, sont dressés, longs de deux centimètres et longuement recourbés en crochets. Le zygophylle pousse dans les régions arides sur des terrains salés (44).

\section{- Symptômes et lésions}

Cette plante, utilisée à des fins thérapeutiques comme remède contre le diabète, provoquerait, d'après les informations fournies par des vétérinaires et des éleveurs de la région de Birine, des avortements chez les brebis. Par ailleurs, les brebis qui ont pâturé sur les terrains où poussait le zygophylle pendant la période de lutte, ont donné naissance à des agneaux normaux mais, au bout de quatre à cinq semaines, ces agneaux ont présenté de l'ataxie, un fléchissement du train postérieur et ne pouvaient rester debout après avoir été relevés. Ces agneaux ne survivent pas en général et, s'ils survivent, leur croissance et leur développement sont médiocres. Comme les brebis qui ont consommé du zygophylle n'ont exhibé aucun symptôme, il est possible que cette plante contienne des principes embryotoxiques.

Drimia maritima (L.) Stearn (scille maritime, scille officinale, urginée maritime, feraoun, beçal el far)

\section{- Caractéristiques}

La scille maritime est une plante de la famille des Liliaceae selon la classification classique, ou des Asparagaceae ou des Hyacinthaceae selon la classification phylogénétique. C'est une plante à bulbe recouvert de tuniques brunes. Les feuilles, lancéolées et multinervées, ont 5 à $15 \mathrm{~cm}$ de large et peuvent atteindre un mètre de long. Elles sont généralement dressées et de couleur vert foncé. Le bulbe est très gros et peut atteindre $20 \mathrm{~cm}$ de diamètre. La tige florifère est très robuste, haute de $40 \mathrm{~cm}$ au moins et peut atteindre 1,5 m. Les fleurs sont disposées en grappe serrée et spiralée, de couleur blanche, assez rarement striées de rose, placées à l'aisselle d'une bractée individuelle (44). Elle est largement répandue en Afrique du Nord et, malgré son nom, elle s'étend profondément à l'intérieur des terres. Les principes toxiques de la scille sont des hétérosides cardiotoniques stéroïdiques dont le scillarène $\mathrm{A}$ et le scillarène B, ainsi que le scilliroside à activité raticide (14).

\section{- Symptômes et lésions}

Les premiers symptômes (inappétence, inquiétude et abattement) apparaissent vingt-quatre heures après ingestion de la plante. Par la suite surviennent des troubles cardiaques et circulatoires, suivis de troubles urinaires avec anurie et parfois hématurie. Après une phase de coma, la mort survient par arrêt cardiaque. Les lésions décrites sont une congestion sous-cutanée, des pétéchies et des suffusions endocardiques, ainsi qu'une gastro-entérite (53). 
La scille maritime a été à l'origine d'une cinquantaine de cas graves rencontrés à l'est de l'Algérie (Annaba) et au nord-ouest (Médéa), où elle serait la première cause de réforme. Les animaux intoxiqués étaient en mauvais état corporel, leur poil était piqué, des tremblements sont apparus, puis ils se sont mis en décubitus et sont morts à moins d'être sacrifiés. Les lésions retrouvées ont été des hémorragies au niveau du tractus digestif. Les symptômes et les lésions décrits par les vétérinaires ont évoqué ceux rapportés par d'autres auteurs $(18,53)$.

\section{Quercus coccifera L. (chêne kermès, ballout)}

\section{- Caractéristiques}

Le chêne kermès, de la famille des Fagaceae, est un arbre ou arbuste à feuilles persistantes et coriaces, d'un à trois mètres de haut et à tiges recouvertes d'une écorce brun noir finement crevassée. Les feuilles ont un aspect luisant sur le dessus et le dessous. Les fleurs jaunâtres apparaissent en avril-mai. Les fruits, isolés, portés par un pédoncule très court, sont des glands, de forme globuleuse. La cupule, qui les enveloppe pour plus de la moitié, est couverte d'écailles rigides, nombreuses, terminées en pointe aiguë. Les chênes contiennent des tanins, substances polyphénoliques dont la structure est voisine de celle des hétérosides (29). L'intoxication survient après consommation de glands ou de jeunes feuilles riches en tanins.

\section{- Symptômes et lésions}

Les signes de l'intoxication apparaissent une dizaine de jours après l'ingestion de glands chez les ruminants. Les premiers signes sont l'anorexie, la prostration, de la polyurie, un œdème ventral, des coliques sourdes, une constipation suivie de diarrhées muqueuses, parfois sanguinolentes (11). Les signes urinaires et hémolytiques sont caractérisés par une douleur aiguë à la palpation de la région lombaire.

Cette intoxication a été décrite par de nombreux vétérinaires à l'est et au nord de l'Algérie, en zone montagneuse. Les symptômes décrits par les vétérinaires ont été des troubles urinaires avec des mictions fréquentes et de l'hématurie. Ces signes urinaires ont également été décrits en France (28). Environ un tiers des animaux intoxiqués sont morts.

\section{Caprins}

Les chèvres sont plus rarement sujettes à des intoxications ; seules deux espèces de plantes ont été incriminées (tableau II).

\section{Hyoscyamus muticus spp. falezlez}

Les chèvres ont semblé moins sensibles que les moutons à cette intoxication. Dans un troupeau comportant 652 moutons et 108 chèvres, seulement 9 chèvres sont mortes $(8$ p. 100) suite à la consommation de la jusquiame, alors que 394 moutons (60 p. 100) en ont été victimes. Les symptômes décrits sont identiques à ceux rapportés chez les ovins.

\section{Nerium oleander L. (laurier rose, defla, ilili)}

\section{- Caractéristiques}

Le laurier rose, de la famille des apocynacées, est un arbuste à tiges érigées, à feuilles coriaces entières, à grandes fleurs roses ou blanches selon les variétés (10). Il renferme un ensemble d'hétérosides de structure proche de celle des hétérosides digitaliques. Dans les feuilles, on trouve principalement l'oléandroside, le nérioside et le nérianthoside parmi les substances responsables de toxicité cardiaque. Toutes les parties de la plante, feuille, fleur, écorce, bois, sont vénéneuses (29).

\section{- Symptômes et lésions}

Les signes de l'intoxication sont cardiaques, gastro-intestinaux et neurologiques. Il y a une bradycardie ou une tachycardie, des arythmies, des blocs auriculo-ventriculaires. On observe des diarrhées, une faiblesse neuro-musculaire et des convulsions. La mort survient 36 heures après l'ingestion (1).

Les cas qui nous ont été rapportés ont concerné des chèvres qui s'étaient abreuvées dans des flaques d'eau, au niveau d'oueds bordés de lauriers roses, dans la région de Ksar-El-Boukhari. Ces animaux ont été retrouvés morts à côté des flaques. Ces observations rejoignent celles décrites par d'autres auteurs $(1,11)$.

\section{Bovins}

Les bovins sont assez souvent concernés par les intoxications végétales ; les plus fréquentes ont été provoquées par neuf espèces (tableau II).

\section{Cistus monspeliensis L. (ciste de Montpellier, oum alyia, tame itibt)}

\section{- Caractéristiques}

Le ciste de Montpellier est un arbuste de la famille des cistacées, haut d'un mètre en moyenne, très ramifié dès la base, velu et visqueux en toutes ses parties. Les feuilles sont lancéolées ou linéaires, et rugueuses. Les fleurs sont blanches, de deux à trois centimètres de diamètre (44). Le ciste de Montpellier est retrouvé dans les forêts et broussailles en terrain non calcaire dans toute l'Algérie.

Il n'existe aucune publication rapportant la toxicité du ciste de Montpellier chez les bovins bien qu'il soit très répandu dans les pays méditerranéens. Toutefois son rôle a été évoqué dans des cas de cystite chez des génisses en transhumance dans le sud de la France (32). En revanche au Maroc, Cistus ladaniferus, C. crispus et $C$. salviifolius sont responsables d'intoxications chez les moutons.

\section{- Symptômes et lésions}

Les symptômes observés sont surtout d'ordre nerveux et digestif. $\mathrm{Au}$ début, l'animal se déplace difficilement, présente une faiblesse générale et une amaurose. Les troubles digestifs apparaissent tardivement avec surtout des coliques et des diarrhées. Des avortements ont été également rapportés chez les femelles gravides (2).

Dans les cas qui nous ont été décrits, une centaine de bovins mâles et femelles, pâturant dans des zones montagneuses à l'est du pays (Annaba), ont présenté des symptômes proches de ceux décrits en France. Selon les éleveurs, cette plante poussant de manière très dense a été consommée quasi exclusivement par les bovins qui n'ont pas trouvé d'autres plantes aussi vertes. Il est à noter qu'après les fréquents incendies des maquis, seul le ciste de Montpellier prolifère. D'après les éleveurs, cette plante n'agirait pas seule mais de manière synergique avec les cendres provenant de cistes calcinés sur lesquelles elle prolifère. Les symptômes décrits ont été l'abattement, l'anorexie, l'hypothermie, la miction difficile, l'oligurie et enfin de l'anurie. Les lésions ont présenté un épaississement des parois vésicales. Aucun signe nerveux n'a été observé.

\section{Ferula communis}

Les cas qui nous ont été décrits ont présenté de l'abattement, de la prostration et de l'épistaxis, ainsi que des diarrhées hémorragiques et une hématurie. A l'examen, les vétérinaires ont observé une tachycardie, une dyspnée et une pâleur des muqueuses. Ces signes cliniques et ces lésions ont également été rapportés par d'autres auteurs $(14,57)$. 
Silybum marianum (L.) Gaertn. (chardon-Marie, chouq boutli, zaz, el bouk)

\section{- Caractéristiques}

Le chardon-Marie est une plante de la famille des astéracées (ou des composées). C'est une plante puissante, généralement bisannuelle, à tiges rameuses et sillonnées, atteignant environ $1,50 \mathrm{~m}$ de haut. Les feuilles sont vertes, maculées de blanc, dentées et épineuses (44). Le chardon-Marie est retrouvé dans les champs, les fossés, les lieux incultes et les décombres. Il serait parmi les plantes les plus riches en nitrates en Israël (47).

\section{- Symptômes et lésions}

Les nitrates qu'il contient sont transformés en nitrites qui provoquent une méthémoglobinisation. La consommation de cette plante en grandes quantités par les bovins est toxique mais, dans de nombreux cas, les bovins peuvent en ingérer beaucoup sans effet visible (11).

L'intoxication qui nous a été rapportée, dans la région de Sedrata à l'est du pays, s'est déclarée en trois jours chez les animaux alimentés exclusivement au chardon-Marie, alors que chez ceux qui recevaient du fourrage et du chardon-Marie, elle ne s'est déclarée qu'au bout de 15 jours. Chez les dix-huit cas rapportés, les symptômes ont été les suivants : troubles neuromusculaires et oculaires se manifestant brusquement, chancellement, difficulté à se tenir debout, tremblements, somnolence, flaccidité musculaire, tachycardie, inappétence et hypothermie. Les muqueuses étaient cyanosées, le sang était brun-noirâtre en raison de la méthémoglobinisation. Les mortalités ont été de 50 p. 100 environ. Etant donné qu'il y a peu de cas décrits d'intoxication par le chardon-Marie, la description des signes cliniques faite par les vétérinaires algériens illustre les effets méthémoglobinisants des nitrates contenus dans cette plante (cyanose, tachycardie, troubles neuromusculaires).

\section{Sorghum drummondii (Stend.) Nees ex Millsp. \& Chase (sorgho)}

\section{- Caractéristiques}

Le sorgho est une plante fourragère de la famille des graminées. Il est utilisé comme culture fourragère d'urgence pour la fin de la saison. Il est consommé soit en pâturage, soit sous forme de fourrage vert. C'est une plante de 1,5 m de haut, dont l'appareil végétatif ressemble à celui du maïs mais, contrairement à ce dernier, il n'y a qu'une inflorescence terminale très fournie et très rameuse de 20 à $40 \mathrm{~cm}$.

Les sorghos sont des plantes cyanogénétiques. Ils renferment de la dhurrine, un glycoside cyanogénétique qui, par hydrolyse, libère l'acide cyanhydrique. La dhurrine, très abondante dans la plante jeune, disparaît progressivement, à mesure que le stade de végétation avance. La libération de l'acide cyanhydrique, qui est volatil, ne se produit que lorsque la plante est endommagée par la gelée, la mastication ou le stress hydrique (25). Des enzymes végétales transforment ce glycoside en libérant de l'acide cyanhydrique.

\section{- Symptômes et lésions}

Si le cyanure se retrouve dans l'organisme de l'animal en quantités suffisantes, il provoque une hypoxie cytotoxique qui peut conduire à la mort par asphyxie. Le sorgho du Soudan peut aussi accumuler des nitrates en quantités toxiques $(15,21,25)$. On peut estimer qu'à la période de toxicité maximale, un kilogramme de feuilles de sorgho suffit pour provoquer une intoxication mortelle chez un bovin de 500 kilogrammes (29).

Dans les cas qui nous ont été rapportés, les vaches sont mortes assez rapidement après avoir pâturé sur des prairies de nouvelles pousses de sorgho. Elles ont présenté une tachypnée, une tachycardie, une démarche chancelante et la présence d'écume à la bouche.
Ce type d'accident est survenu chez les éleveurs ayant introduit cette culture fourragère estivale en méconnaissant le danger des jeunes plantes riches en dhurrine. Les symptômes rapportés chez des bovins en France sont une démarche chancelante, une dyspnée et des convulsions (29).

\section{Pteridium aquilinum (L.) Kuhn (fougère aigle,fersiou,fersiq)}

\section{- Caractéristiques}

La fougère aigle est une plante de la famille des Dennstaedtiaceae, haute de 0,40 à 2,50 m. Les frondes, issues d'un rhizome robuste, sortent de terre isolément. Elles sont profondément découpées avec deux ordres de ramification $(14,29)$. Les frondes et le rhizome contiennent un principe toxique cancérigène qui est un hétéroside norsesquiterpénique, le ptaquiloside. Cette molécule très labile se transforme en milieu alcalin en une diénone réactive qui peut s'additionner, via une carbocation électrophile, sur l'ADN et devenir mutagène et cancérigène (43). Il existe également une thiaminase très active mais qui n'intervient pas dans la genèse de l'intoxication des bovins $(28,57)$.

\section{- Symptômes et lésions}

L'intoxication aiguë se manifeste par un syndrome hémorragique avec de l'hyperthermie, de la faiblesse, de l'anorexie, de l'épistaxis, de l'hématurie et parfois de l'hématidrose (sueurs de sang). Ces lésions sont liées à une atteinte de la moelle osseuse induisant une leucopénie et une thrombocytopénie $(30,57)$. Dans l'intoxication chronique on observe une hématurie consécutive à un saignement de la muqueuse vésicale. Des adénomes, des tumeurs épithéliales ainsi que des hémangiomes sont retrouvés dans la vessie $(23,28,40)$.

Sur une dizaine de cas rapportés par les vétérinaires des régions montagneuses de Kabylie où la fougère aigle est prépondérante, les symptômes observés - hématidrose (sueurs de sang) et hématurie - ont été identiques à ceux décrits en France (28). Les animaux intoxiqués ont été abattus avant l'issue mortelle.

\section{Malva parviflora L. (mauve, khobaï, medjir)}

\section{- Caractéristiques}

La mauve est une plante bisannuelle ou vivace, de la famille des malvacées, à tiges ramifiées, pouvant atteindre environ un mètre de haut. Les fleurs pédonculées groupées à plusieurs à l'aisselle des feuilles sont de couleur rose violacé (44). La mauve est responsable d'intoxications chez les moutons et les chevaux en Israël et en Australie. Comme le chardon-Marie, elle serait parmi les plantes les plus riches en nitrates en Israël (47).

\section{- Symptômes et lésions}

Les symptômes décrits par les vétérinaires de différentes régions sont des tremblements musculaires, de la faiblesse et un décubitus chez les moutons après consommation de grandes quantités de plantes pendant plusieurs jours. Ces symptômes ont également été rapportés par d'autres auteurs $(11,39,47)$ qui ont cependant relevé de faibles mortalités.

\section{Oxalis pes-caprae L. (oxalis, surelle, qouarça, qrioussa)}

\section{- Caractéristiques}

L'oxalis est une plante vivace de la famille des oxalidacées, haute de 10 à 25 centimètres, ses feuilles sont longuement pétiolées, et les fleurs sont jaunes (44). Les intoxications par l'oxalis sont assez fréquentes dans les régions méditerranéennes et surviennent surtout à la fin de l'automne et pendant l'hiver $(45,48)$. L'oxalis contient de l'acide oxalique qui, par réaction avec le calcium et le magnésium sanguins, forme des oxalates insolubles. Ces oxalates sont des poisons systémiques et corrosifs pour les reins. 


\section{- Symptômes et lésions}

Les oxalates inhibent la motricité du rumen entraînant une météorisation. L'ingestion de quantités importantes d'oxalis entraîne une hypocalcémie sévère, une dépression du système nerveux central et la mort survient par un collapsus cardiovasculaire. Dans les cas chroniques, l'hypocalcémie est compliquée par une urolithiase et une élévation de l'urémie (20).

Les vétérinaires de différentes régions du Nord rapportent de nombreux cas d'hypocalcémie avec dépression du système nerveux central et syndrome de la vache couchée dus à l'ingestion élevée d'oxalis. Ces signes cliniques sont également rapportés en Tunisie où Frikha a observé dans les cas chroniques une hypocalcémie compliquée par une urolithiase et une élévation de l'urémie (20).

\section{Drimia maritima}

La scille maritime est à l'origine d'une trentaine de cas graves rencontrés à l'est de l'Algérie (Annaba) et au nord-ouest (Médéa), où elle serait la première cause de réforme. Les animaux intoxiqués par cette plante étaient en mauvais état corporel, leur poil était piqué, des tremblements sont apparus, puis ils se sont mis en décubitus et sont morts, à moins d'être sacrifiés. Ces symptômes et ces lésions décrits par les vétérinaires correspondaient à ceux déjà rapportés par d'autres auteurs $(18,53)$.

\section{Quercus coccifera}

Les signes décrits sont des troubles urinaires avec hématurie. Les vétérinaires ont noté que les animaux ayant consommé de grandes quantités de glands et de feuilles de chêne étaient très amaigris, avaient un poil piqué et semblaient déprimés. Leur peau prenait l'aspect d'une carapace de tortue et ils émettaient un son de tambour en se déplaçant. Un tiers des animaux sont morts. Ces troubles urinaires avec hématurie ont également été décrits par plusieurs auteurs $(22,31,46)$.

\section{Dromadaires}

Six plantes toxiques ont été le plus souvent incriminées.

\section{Hyoscyamus muticus spp.falezlez}

Des dromadaires ayant consommé de la jusquiame ont présenté des troubles nerveux avec agressivité mais aucune mortalité n'a été rapportée.

\section{Sorghum halepense (L.) Pers. (sorgho d'Alep, dokna)}

- Caractéristiques

Le sorgho d'Alep est une plante vivace, de la famille des graminées à rhizome rampant et blanc nacré, qui atteint $1,50 \mathrm{~m}$ de haut et qui pousse comme une mauvaise herbe. Comme les autres variétés de sorgho, il contient des hétérosides cyanogénétiques. Il peut produire de l'acide cyanhydrique en quantités toxiques s'il est endommagé par la mastication ou le stress hydrique.

\section{- Symptômes et lésions}

Des dromadaires ayant consommé cette plante en sont morts assez brutalement. Les symptômes décrits étaient une accélération de la respiration, un pouls irrégulier, la présence d'écume à la bouche et une démarche chancelante.

\section{Calobota saharae (genêt saharien, tellegit, mukh)}

\section{- Caractéristiques}

Le genêt saharien est une plante de la famille des fabacées, à longs rameaux cylindriques et érigés, d'un à deux mètres de haut. Il s'agit d'une espèce saharienne, poussant dans les rocailles plus ou moins ensablées (44). Ses principes toxiques ne sont pas connus, en revanche, ceux du genêt à balais (Cytisus scoparius) et du genêt d'Espagne (Spartium junceum L.) le sont : les rameaux du genêt à balais renferment de la spartéine ganglioplégique et ceux du genêt d'Espagne de la cytisine (14).

- Symptômes et lésions

Les vétérinaires ont rapporté que les dromadaires ayant ingéré du genêt saharien sur une longue période sont devenus apathiques et ont eu des difficultés de miction du fait de la présence d'un bouchon blanchâtre dans l'urètre.

\section{Malva parviflora}

La consommation excessive de mauve a provoqué chez les dromadaires des troubles nerveux avec paralysies et œè̀mes des membres.

\section{Thapsia garganica}

La consommation de cette plante vésicante par les dromadaires a provoqué des signes de violente gastro-entérite.

\section{Diplotaxis harra (Forssk.) Boiss. (mazloum, lechnef)}

- Caractéristiques

Diplotaxis harra est une plante de la famille des Brassicaceae (encore appelée crucifères) à tiges florifères feuillées, au moins à la base (44). Les plantes de cette famille élaborent des glucosinolates qui sont des molécules soufrées responsables des odeurs fortes caractéristiques de ces végétaux (14). Les Brassicaceae peuvent contenir, dans certaines conditions, des nitriles (26).

\section{- Symptômes et lésions}

Chez les bovins, les plantes de la famille des Brassicaceae entraînent divers syndromes avec anémie hémolytique, cécité, emphysème pulmonaire et troubles digestifs, qui peuvent se produire séparément ou en association. Blood et Henderson signalent également des cas d'intoxication par les nitrates et les nitrites (11).

Selon les vétérinaires qui ont décrit cette intoxication, cette crucifère ne serait toxique qu'à partir de la deuxième année de végétation. Sa consommation par les dromadaires dans le Sud algérien a provoqué des paralysies et les animaux ont alors renâclé à se relever.

\section{CONCLUSION}

Les intoxications par les plantes sont suffisamment répandues pour nécessiter la diffusion des informations nécessaires tant auprès des vétérinaires que des éleveurs. De nombreuses intoxications pourraient être évitées si certaines précautions étaient prises. Ainsi, comme la plupart des intoxications concernent surtout les moutons, la connaissance de la flore permettrait de limiter ce genre d'accident, notamment en cas de mobilité des troupeaux vers des pâturages à la composition floristique différente et peu connue des bêtes et des pasteurs. Certaines zones où prolifèrent des plantes toxiques sont évitées par les éleveurs de la région qui les reconnaissent comme étant dangereuses, alors qu'elles pourraient paraître attrayantes pour des troupeaux de passage qui ne les connaissent pas. De plus, alors que le dessèchement de la flore sur les lieux de pâture en saison sèche est presque total, beaucoup de plantes toxiques conservent leur verdure, attirant les animaux en quête de plantes vertes. Les animaux en état de sous-nutrition ou présentant une dépravation du goût s'intoxiquent facilement.

La toxicité d'une plante pouvant varier en fonction de son stade de développement et de la saison, la connaissance de cette variabilité 
doit s'ajouter à l'identification des plantes toxiques elles-mêmes. Par ailleurs, certaines plantes ne se développant qu'au cours des années à pluviométrie abondante, leur présence aléatoire et parfois éphémère accentue le risque d'intoxication.

Dans ce contexte où la toxicité d'une plante n'est pas toujours clairement établie, la toxicologie vétérinaire se doit d'approfondir certains travaux visant à mettre en place des expérimentations afin de reproduire les troubles observés sur le terrain selon la plante incriminée.

\section{BIBLIOGRAPHIE}

1. ADAM S.E.I., AL-YAHYA M.A., AL-FARHAN A.H., 2001. Acute toxicity of various oral doses of dried Nerium oleander leaves in sheep. Am. J. Chin. Med., 29: 525-532.

2. ALAOUI J., 1981. Contribution à l'étude des principales plantes toxiques chez les animaux domestiques au Maroc. Thèse Doct. Vét. Institut agronomique et vétérinaire Hassan II, Rabat, Maroc.

3. ALI H., BROGGER CHRISTENSEN S., FOREMAN I.C., PEARCE F.L., PIOTROWSKI W., THASTRUP O., 1985. The ability of thapsigargin and thapsigargicin to activate cells involved in the inflammatory response. $\mathrm{Br}$. J. Pharmacol., 85: 705-712.

4. APPENDINO G., TAGLIAPETRA S., GARIBOLDI P., NANO G.M. PICCI V., 1988. $\Omega$-oxygenated prenylated coumarins from Ferula communis. Phytochemistry, 27: 3619-3624.

5. ARAGNO M., TAGLIAPIETRA S., NANO G.M., UGAZIO G., 1989 Experimental studies on the toxicity of some compounds from Ferula communis in the rat. Res. Commun. Chem. Pathol. Pharmacol., 66: 333336.

6. ARAYA O.S., FORD E.J., 1981. An investigation of the type of photosensitization caused by the ingestion of St John's wort (Hypericum perforatum) by calves. J. Comp. Pathol., 91: 135-141.

7. ASLANI M.R., REZAKHANI A., 2000. A case report of oleander (Nerium oleander) intoxication in cattle. Int. J. Trop. Agric., 18: 185-187.

8. BATANOUNY K.H., 1999. Wild medicinal plants in Egypt: an inventory to support conservation and sustainable use. Cairo, Egypt, Palm Press, $207 \mathrm{p}$.

9. BEIN C., GRANIER M., ELCADI T., DELACOUR J.L., WAGSCHAL G., 1999. Une cause rare de mydriase unilatérale. Presse Méd., 28 : 1070.

10. BEZANGER-BEAUQUESNE L., PINKAS M., TORCK M., TROTIN F., 1980. Plantes médicinales des régions tempérées. Paris, France, Maloine, $439 \mathrm{p}$.

11. BLOOD D.C., HENDERSON J.A., 1976. Médecine vétérinaire, $2^{e}$ édn. Paris, France, Vigot Frères, $992 \mathrm{p}$.

12. BREMOND G., 1887. Journal de l'Ecole de Lyon (cité par Velu et Gardas, 1924).

13. BRUNETON J., 1999. Pharmacognosy, phytochemistry, medicinal plants, 2nd Edn. Paris, France, Lavoisier, 1119 p.

14. BRUNETON J., 2005. Plantes toxiques et végétaux dangereux pour I'homme et les animaux, $3^{\mathrm{e}}$ édn. Paris, France, Lavoisier, $618 \mathrm{p}$.

15. CLAY B.R., EDWARDS W.C., PETERSON D.R., 1976. Toxic nitrate accumulation in the sorghums. Bovine Pract., 11: 28-32.

16. COOPER M.R., JOHNSON A.W., 1984. Poisonous plants in Britain and their effects on animals and man. London, UK, Her Majesty's Stationery Office, 305 p.

17. DREW D.P., RASMUSSEN S.K., AVATO P., SIMONSEN H.T., 2012 A comparison of headspace solid-phase microextraction and classic hydrodistillation for the identification of volatile constituents from Thapsia spp. provides insights into guaianolide biosynthesis in Apiaceae. Phytochem. Anal., 23: 44-51.

18. EL BAHRI L., DJEGHAM M., MAKHLOUF M., 2000. Urginea maritima L. (Squill): A poisonous plant of North Africa. Vet. Hum. Toxicol., 42: 108-110.

19. EL BAHRI L., MAKHLOUF M., 2001. Thapsia garganica L.: A poisonous plant of North Africa. Vet. Hum. Toxicol., 43: 216-218.

20. FRIKHA M., 1980. Toxicologie des oxalates : contribution à l'étude de l'intoxication par l'oxalis chez les ruminants en Tunisie. Thèse Doct. Vét., Ecole nationale de médecine vétérinaire, Sidi Thabet, Tunisie.
21. FULLER T.C., MCCLINTOCK E., 1986. Poisonous plants of California. Berkeley, USA, University of California Press, $432 \mathrm{p}$.

22. GARG S.K., MAKKAR H.P.S., NAGAL K.B., SHARMA S.K., WADHWA D.R., SINGH B., 1992. Oak (Quercus incana) leaf poisoning in cattle. Vet. Hum. Toxicol., 34: 161-164.

23. GAVA A., SILVA NEVES D., GAVA D., DE MOURA S.T., SCHILD A.L, RIET-CORREA F., 2002. Bracken fern (Pteridium aquilinum) poisoning in cattle in Southern Brazil. Vet. Hum. Toxicol., 44: 362-365.

24. GIL M.C., GOMEZ L., ROY T.J., PRIETO L., PENA F.J., GARCIA L., 2002. Testicular and epididymal changes in rams following intoxication by Ferula communis. Vet. Rec., 150: 24-25.

25. GRAY E., RICE J.S., WATTENBARGER D., BENSON J.A., HESTER A.J., LOYD R.C., GREENE B.M., 1968. Hydrocyanic acid potential of Sorghum plants grown in Tennessee. University of Tennessee Agricultural Experiment Station Bulletin AgResearch: 1-48.

26. HALKIER B.A., 1999. Glucosinolates. In: Ikan R., Ed., Naturally occurring glycosides. Chichester, UK, John Wiley, p. 193-223.

27. HUGNET C., GAULT G., BERNY P., LORGUE G., 1995. Intoxication par les hétérosides cardiotoniques d'origine végétale. Rec. Méd. Vét., $171: 177-181$

28. JEAN-BLAIN C., GASTELLU J., BRINGUIER P.P., 1987. Hématurie chronique des bovins : étude clinique. Point Vét., 19 : 317-323.

29. JEAN-BLAIN C., GRISVARD M., 1973. Plantes vénéneuses et toxicologie. Paris, France, La maison rustique, $140 \mathrm{p}$.

30. HIRONO I., KONO Y., TAKAHASHI K., 1984. Reproduction of acute bracken poisoning in a calf with ptaquiloside, a bracken constituent. Vet. Rec., 115: 375-378.

31. HOLLIMAN A., 1985. Acorn poisoning in ruminants. Vet. Rec., 116: 546 .

32. KECK G., BEZILLE P., GAUT G., SIMON P., RAFFIN Y., CARAGUEL B., 1998. Cystite d'origine toxique probable : première observation chez des génisses transhumantes en milieu méditerranéen. Point Vet., 29 : 1347-1349.

33. KUSARI S., LAMSHOFT M., ZUHLKE S., SPITELLER M., 2008. An endophytic fungus from Hypericum perforatum that produces hypericin. J. Nat. Prod., 71: 159-162.

34. LAMNAOUER D., 1999. Activité anticoagulante des coumarines de Ferula communis L. Thérapie, 54 : 747-751.

35. LAMNAOUER D., BODO B., MARTIN M.T., MOLHO D., 1987. Ferulenol and 20-hydroxyferulenol, toxic coumarins from Ferula communis var. genuina. Phytochemistry, 26: 1613.

36. LAMNAOUER D., FRAIGUI O., 1991. Structure of isoferprenin, a 4-hydroxycoumarin derivative from Ferula communis. var. genuina. J. Nat. Prod., 54: 576-578.

37. LAMNAOUER D., FRAIGUI O., ABADOME F., 1991. Toxicité et activité anticoagulante de quelques constituants de Ferula communis L. chez le rat, Al Biruniya. Rev. Mar. Pharm., 7 : 135-142.

38. LAMNAOUER D., FRAIGUI O., MARTIN M.T., BODO B., 1991. Structure of ferulenol derivatives from Ferula communis var. genuina. Phytochemistry, 30: 2383-2386.

39. MAIN D.C., BUTLER R.A., 2006. Probable Malva parviflora (small flowered mallow) intoxication in sheep in Western Australia. Aust. Vet. J., 84: $134-135$

40. MARRERO E., BULNES C., SANCHEZ L.M., PALENZUELA I., STUART R., JACOBS F., ROMERO J., 2001. Pteridium aquilinum (bracken fern) toxicity in cattle in the humid chaco of Tarija, Bolivia. Vet. Hum. Toxicol., 43: 156-158.

41. MEYER C., éd., 2015. Dictionnaire des sciences animales. Montpellier, France, Cirad. http://dico-sciences-animales.cirad.fr/

42. MOHAMMEDI D., LAMNAOUER D., 2005. Chimiotaxonomie de Ferula communis L. en Algérie. In : Congrès international sur les plantes médicinales, Errachidia, Maroc, 16-19 mars 2005.

43. OJIKA M., WAKAMATSU K., NIWA H., YAMADA K., 1987. Ptaquiloside, a potent carcinogen isolated from bracken fern Pteridium aquilinum var. latiusculum: Structure elucidation based on chemical and spectral evidence, and reactions with amino acids, nucleotides and nucleosides. Tetrahedron, 43: 5261-5264.

44. QUEZEL P., SANTA S., 1962, 1963. Nouvelle flore de I'Algérie. Paris, France, CNRS, $1170 \mathrm{p}$ 
45. REKHIS J., 1994. The poisonous plants Oxalis cernua. Vet. Hum. Toxicol., 36: 36-38.

46. SAVEY M., 1985. Diagnostic d'une intoxication par les glands. Point Vét., $17: 365-366$

47. SHLOSBERG A., 2011. Plant toxicoses in Israel. Israel J. Vet. Med., 66: $171-174$.

48. SHLOSBERG A., EGYED M.N., 1983. Examples of poisonous plants in Israel of importance to animals and man. Arch. Toxicol., 6: 194S-196S.

49. SHLOSBERG A., EGYED M.N., 1985. Experimental Ferula communis (giant fennel toxicosis in sheep. Zentralb/. Veterinarmed. A, 32: 778-784.

50. SIJILMASSI A., 1996. Les plantes médicinales du Maroc, $6^{\mathrm{e}}$ édn. Casablanca, Maroc, Fennec, 285 p.

51. TERESA J. DE P, VILLASECO M.A, HERNANDEZ J.M, MORAN J.R. URONES J.G., GRANDE M., 1986. Complex acetylenes from the roots of Ferula communis. Planta Med., 6: 458-462.

52. THEODOSSIOU T.A., NORONHA-DUTRA A., HOTHARSALL J.S., 2006. Mitochondria are a primary target of hypericin phototoxicity: synergy of intracellular calcium mobilisation in cell killing. Int. J. Biochem. Cell Biol., 38: 1946-1956.

53. TUNCOK Y., KOZAN O., CAVDAR C., GUVEN H., FOWLER J., 1995. Urginea maritima L. (Squill) toxicity. J. Toxicol. Clin. Toxicol., 33: 83-86.

54. VALLE M.G., APPENDINO G., NANO G.M., PICCI V., 1987. Prenylated coumarins and sesquiterpenoids from Ferula communis. Phytochemistry, 26: 253-256.

55. VELU H., GARDAS J., 1924. Le férulisme. Arch. Inst. Pasteur, Alger, 2 : 494-505.

56. VIALA A., 1998. Les toxicomanies : solanacées et dérivés. In : Eléments de toxicologie. Paris, France, Editions médicales internationales, p. 426-427.

57. XU L.R, 1992. Bracken poisoning and enzootic haematuria in cattle in China. Res. Vet. Sci., 53: 116-121.

Accepted 27 March 2015; Online publication 30 September 2015

\section{Summary}

Mohammedi D., Mohammedi S., Keck G. Major plant poisonings in ruminants in the Mediterranean area

Plant poisonings in ruminants are rather common, but they remain little known and difficult to quantify because of the diversity of the toxic plants responsible for them. The impacts of plant poisoning in the Mediterranean Basin should be better evaluated to distinguish them from infectious origins. Indeed, veterinarians find it difficult to reach a diagnosis of plant poisoning and often incriminate infectious agents. It is therefore important to gather plenty of objective information, which often proves difficult given the approximative case descriptions. Some of these toxic plants are very widely distributed south and north of the Mediterranean, including in France. In contrast, others are relatively specific to the desert environment of North Africa and are present in particular in Algeria. The most frequently affected animals were sheep $(61.5 \%)$, cattle $(24.7 \%)$, camels $(9 \%)$ and goats $(4.6 \%)$. Poisonings encountered in ruminants were caused by Hyoscyamus muticus spp. falezlez (Coss.) Maire, Ferula communis L., Thapsia garganica, Hypericum crispum L., Tetraena cornuta (Coss.) Beier \& Thulin, Drimia maritima (L.) Stearn, Quercus coccifera L., Nerium oleander L., Cistus monspeliensis L., Silybum marianum (L.) Gaertn., Sorghum drummondii (Stend.) Nees ex Millsp. \& Chase, Pteridium aquilinum (L.) Kuhn, Malva parviflora L., Oxalis pes-caprae L., Sorghum halepense, Calobota saharae, Diplotaxis harra (Forssk.) Boiss.

Keywords: Ruminant - Poisoning - Hyoscyamus muticus Ferula communis - Quercus coccifera - Cistus monspeliensis Silybum marianum - Algeria.

\section{Resumen}

Mohammedi D., Mohammedi S., Keck G. Principales intoxicaciones vegetales en los rumiantes en zona mediterránea

Las intoxicaciones vegetales en los rumiantes son bastante frecuentes, pero aún mal conocidas y su importancia difícil de cuantificar, debido al hecho de la diversidad de las plantas tóxicas responsables. Los impactos de las intoxicaciones vegetales en la Cuenca mediterránea deberían ser mejor evaluados para distinguirlos de los causados por infecciones. En efecto, los veterinarios establecen difícilmente un diagnóstico de intoxicación vegetal e incriminan a menudo a agentes infecciosos. Es por lo tanto importante reunir un máximo de información objetiva, lo que es a menudo delicado en razón de las informaciones relativamente aproximativas en las descripciones de los casos. Ciertas plantas responsables de intoxicación presentan una distribución muy amplia en el sur y en el norte del Mediterráneo, incluyendo Francia. Otras, por el contrario, son relativamente específicas a los medios desérticos del Magreb y principalmente Argelia. Los animales más frecuentemente concernidos son los ovinos $(61,5 \%)$, los bovinos $(24,7 \%)$, los dromedarios $(9 \%)$ y los caprinos $(4,6 \%)$ Las intoxicaciones encontradas en los rumiantes son provocadas por Hyoscyamus muticus spp. falezlez (Coss.) Maire, Ferula communis L., Thapsia garganica, Hypericum crispum L., Tetraena cornuta (Coss.) Beier \& Thulin, Drimia maritima (L.) Stearn, Quercus coccifera L., Nerium oleander L., Cistus monspeliensis L., Silybum marianum (L.) Gaertn., Sorghum drummondii (Stend.) Nees ex Millsp. \& Chase, Pteridium aquilinum (L.) Kuhn, Malva parviflora L., Oxalis pes-caprae L., Sorghum halepense, Calobota saharae, Diplotaxis harra (Forssk.) Boiss.

Palabras clave: Rumiante - Envenenamiento - Hyoscyamus muticus - Ferula communis - Quercus coccifera - Cistus monspeliensis - Silybum marianum - Argelia. 
